hep-th/9207118 USC-92/HEP-B4

July 1992

\title{
HETEROTIC STRING MODELS IN CURVED SPACETIME *
}

\author{
ITZHAK BARS \\ Physics Department \\ University of Southern California \\ Los Angeles, CA 90089-0484, USA
}

\begin{abstract}
We explore the possibility of string theories in only four spacetime dimensions without any additional compactified dimensions. We show that, provided the theory is defined in curved spacetime that has a cosmological interpration, it is possible to construct consistent heterotic string theories based on a few non-compact current algebra cosets. We classify these models. The gauge groups that emerge fall within a remarkably narrow range and include the desirable low energy flavor symmetry of $S U(3) \times S U(2) \times U(1)$. The quark and lepton states, which come in color triplets and $S U(2)$ doublets, are expected to emerge in several families.
\end{abstract}

\footnotetext{
* Research supported in part by DOE, under Grant No. DE-FG03-84ER-40168
} 


\section{Introduction}

During the past year there has been considerable interest in strings propagating in curved spacetime backgrounds. This was spurred by the fact that some such models can be formulated as conformally exact current algebra coset models, or equivalently as gauged WZW models, based on non-compact groups [1], and that their geometry describes gravitational singularities of both black hole [2] and cosmological types. Before we begin the main technical part of this paper it is appropriate to make some remarks on why it is interesting to further study such models. Of course, they provide a setting for investigating the very interesting problem of gravitational singularities, but is there more?

From experience with string theory we have learned that a conformal field theory that is a candidate for a "classical" string vacuum may be expected to describe the physics at Planck scales. It has been popular to make the assumption that the string vacuum is flat in four Minkowski dimensions and that there are additional compactified "internal" dimensions. In the past all low energy model building efforts have been based on this unjustified assumption. Although initially it appeared very promising, the later discovery of hundreds of thousands of "vacuua" have diminished the confidence of model builders. It must be noticed that the multitude of string vacuua occur in the extra dimensions.

Of course, it was not necessary to assume that the first four dimensions are flat. Instead, one could imagine a cosmological scenario in which the four dimensions evolve toward flat spacetime as a function of time. Furthermore, it was not necessary to assume that there are more than four dimensions. Recall that extra dimensions appeared historically because the mathematical consistency of flat strings required it. But in curved spacetime, conformal invariance can perfectly well be satisfied in any dimension, as the non-compact coset models have demonstrated (even just two dimensions is mathematically consistent). Therefore, it is conceivable that there are no extra dimensions at all.

String theory is needed to describe physics at very early times or very short distances near the Planck length. Let us consider a sigma model formalism which provides a glimpse of the geometry at short distances. Which features of this geometry can be extrapolated to larger distances? Since there are a few phase transitions that must be taken into account it is important to distinguish the features that are likely to be different at large distances after the phase transitions. First, there is the dilaton which starts out massless. Since it should not spoil the long range gravitational forces, it must get a mass near the Planck scale through a phase transition. So far very little effort has been put into this issue, and 
it remains as one of the challenges for string theory. Perhaps this requires understanding non-perturbative effects. Unfortunately, the present state of affairs allows us to hide many problems behind this unresolved point. Next, from experience with grand unified theories one also knows that phase transitions associated with gauge forces, through the mechanism of inflation, can explain why the universe is homogeneous and isotropic.

So, the universe (as described by the sigma model metric) need not start out homogeneous and isotropic or flat in four dimensions. It would be sufficient to start out with a part of the universe which is expanding in four dimensions, and that by the time its size reaches a few Planck scales it approaches a flat universe. If this part of the universe undergoes inflation it may describe our observed universe. The background geometries provided by the non-compact coset models include such geometries in $3 \mathrm{~d}$ and $4 \mathrm{~d}$ (see e.g. [3] [4]). In addition, heterotic string models with such backgrounds predict gauge fields and a spectrum of matter that provides candidates for the low energy quarks and leptons. We know that the forces associated with gauge fields and self couplings of matter could explain the mechanism for mass generation. So, we may defer the mass generation problem to energies well below the Planck scale.

Therefore we may consider a scenario in which there are only three space and one time dimensions. Then the conformal string theory must be in curved spacetime and is designed to satisfy the conditions of exact conformal invariance. The geometry at the Planck length is not necessarily homogeneous or isotropic. At least some bundle of geodesics (that represent the early evolution of part of the universe that gets later inflated) migrate to regions of flat spacetime within a few units of Planck time, perhaps exponentially (as in the $d=3,4$ non-compact models [3] [4] ). The gauge and matter fields of the heterotic theory can survive to low energies through the mechanism of gauge symmetries and chiral symmetries. Some of this "low energy matter" will become all of the matter in the inflated universe. Therefore, such a heterotic string theory can be used to at least classify the particles in multiplets of the symmetry group and compare to the known low energy classification of quarks and leptons.

The initiation of such a program is one of the purposes of the present paper. We will classify the heterotic string models in just four dimensions that can be constructed as exact conformal theories based on non-compact groups. We find that the list of such models is rather short. We will be able to extract the gauge symmetry content of these models and show that the possible gauge groups fall within a remarkably narrow range, and always include the desirable low energy symmetries. This approach does not explain 
why we live in four dimensions, and of course the program can be carried out also with additional compact dimensions. But it seems very interesting to find out what kinds of results emerge if there are in fact only four dimensions.

There exists by now a few models of strings propagating in curved spacetime that are in principle solvable due to the fact that they are formulated as non-compact current algebra cosets based on non-compact groups. The classification of the cosets $G / H$ that yield a single time coordinate [1] is known [5]. The cosets that lead to models in four curved spacetime dimensions $(D=4)$ always include $S O(d-1,2) / S O(d-1,1)$ for $d \leq 4$. In this paper we will assume that there are no more than $D=4$ dimensions and therefore use only $S O(d-1,2)$ for $d=2,3,4$. For $D=d=4$ there are no other bosonic coordinates. When $d \leq 3$, then $D-d=4-d$ additional bosonic coordinates are supplied by taking direct products with other groups (including space-like $U(1)$ or $\mathbb{R}$ factors) and then gauging an appropriate subgroup. Furthermore, we include in our list the possibility of a time-like bosonic coordinate and denote it by a factor of $T$ instead of $\mathbb{R}$. All possibilities are listed in Table-1 in the column labelled "right movers".

\begin{tabular}{|c|c|c|}
\hline$\#$ & left movers with $\mathrm{N}=1 \mathrm{SUSY}$ & right movers \\
\hline 1 & $S O(3,2)_{-k} \times S O(3,1)_{1} / S O(3,1)_{-k+1}$ & $S O(3,2)_{-k} / S O(3,1)_{-k}$ \\
\hline 2 & $\frac{S L(2, \mathbb{R})_{-k_{1}} \times S L(2, \mathbb{R})_{-k_{2}} \times S O(3,1)_{1}}{S L(2, \mathbb{R})_{-k_{1}}-k_{2}+2} \times \mathbb{R}$ & $\frac{S L(2, \mathbb{R})_{-k_{1}} \times S L(2, \mathbb{R})_{-k_{2}}}{S L(2, \mathbb{R})_{-k_{1}}-k_{2}} \times \mathbb{R}$ \\
\hline 3 & $\left(S O(2,2)_{-k} \times S O(3,1)_{1} / S O(2,1)_{-k+2}\right) \times \mathbb{R}$ & $\left(S O(2,2)_{-k} / S O(2,1)_{-k}\right) \times \mathbb{R}$ \\
\hline 4 & $S L(2, \mathbb{R})_{-k} \times S O(3,1)_{1} \times \mathbb{R}$ & $S L(2, \mathbb{R})_{-k} \times \mathbb{R}$ \\
\hline 5 & $\frac{S L(2, \mathbb{R})_{-k_{1}} \times S L(2, \mathbb{R})_{-k_{2}} \times S O(3,1)_{1}}{T \times \mathbb{R}}$ & $S L(2, \mathbb{R})_{-k_{1}} \times S L(2, \mathbb{R})_{-k_{2}} /(T \times \mathbb{R})$ \\
\hline 6 & $S L(2, \mathbb{R})_{-k_{1}} \times S U(2)_{k_{2}} \times S O(3,1)_{1} / \mathbb{R}^{2}$ & $S L(2, \mathbb{R})_{-k_{1}} \times S U(2)_{k_{2}} / \mathbb{R}^{2}$ \\
\hline 7 & $\left(S L(2, \mathbb{R})_{-k} \times \mathbb{R}^{2} \times S O(3,1)_{1}\right) / \mathbb{R}$ & $\left(S L(2, \mathbb{R})_{-k} \times \mathbb{R}^{2}\right) / \mathbb{R}$ \\
\hline 8 & $T \times \mathbb{R}^{3} \times S O(3,1)_{1}$ & $T \times \mathbb{R}^{3}$ \\
\hline 9 & $T \times \frac{S U(2)_{k_{1}} \times S U(2)_{k_{2}} \times S O(3,1)_{1}}{S U(2)_{k_{1}+k_{2}+2}}$ & $T \times \frac{S U(2)_{k_{1}} \times S U(2)_{k_{2}}}{S U(2)_{k_{1}+k_{2}}}$ \\
\hline 10 & $T \times S O(4)_{k} \times S O(3,1)_{1} / S U(2)_{k+2}$ & $T \times S O(4)_{k} / S U(2)_{k}$ \\
\hline 11 & $T \times S U(2)_{k} \times S O(3,1)_{1}$ & $T \times S U(2)_{k}$ \\
\hline 12 & $\left(T \times \mathbb{R} \times S U(2)_{k} \times S O(3,1)_{1}\right) / \mathbb{R}$ & $\left(T \times \mathbb{R} \times S U(2)_{k}\right) / \mathbb{R}$ \\
\hline 13 & $\left(T \times \mathbb{R} \times S L(2, \mathbb{R})_{-k} \times S O(3,1)_{1}\right) / T$ & $\left(T \times \mathbb{R} \times S L(2, R)_{-k}\right) / T$ \\
\hline
\end{tabular}

For brevity we used $\mathbb{R}$ where we could have used either $\mathbb{R}$ or $U(1)$. Case 3 is obtained from case 2 in the limit $k_{1}=k_{2}=k$, while case 4 is the $k_{1}=k, k_{2}=\infty$ limit of either case 
2 or 5 . Similarly, cases 10,11 are limits of case 9 . Furthermore, case 8 may be considered the large $k$ limit of case 11. This last case has unique properties in that its geometry is flat, homogeneous and isotropic (modulo boundary conditions on the $\mathbb{R}^{3}$ factor). One may also notice that cases 9-13 are analytic continuations of cases 3-7. We have listed all these limits or analytic continuations separately because they lead to different gauge groups as will be seen in Table-2 below.

For the numerator factor $T$ in cases 8-13 we allow a background charge $Q_{0}$. The background charge for the time-like coordinate contributes $c_{T}=\left(1+12 Q_{0}^{2}\right)$ to the Virasoro central charge, and this quantitity is always larger than one. Similarly, every space-like coordinate associated with the factors of $\mathbb{R}$ in the numerators may be allowed to have a nontrivial background charge $Q$. This contributes $c_{\mathbb{R}}=\left(1-12 Q^{2}\right)$ for a space-like coordinate, and is always less than one and positive. In the following, to keep our expressions simple, we will assume that $Q=0$. A non-zero $Q$ makes no difference for the discussion below, but we will indicate separately the changes that occur at intermediate steps.

The cases $5,6,7,12,13$ which contain a $T$ or $\mathbb{R}$ factor in the denominator may further be generalized by multiplying both numerator and denominator by a factor $\mathbb{R}^{n}$. What this implies is that there are many possible ways of gauging the $\mathbb{R}$ and/or $T$ factors by taking linear combinations. This may lead to models that are different, however this generalization does not change the results given in Table-2 at all.

The heterotic string will have a supersymmetric left-moving sector and a nonsupersymmetric right-moving sector. The cosets above describe the four dimensional space-time part of the right-moving sector. This contributes $c_{R}(4 D)$ toward the Virasoro central charge. After we analyse the central charge of the supersymmetric left movers and fix it to be $c_{L}=15$ in only four dimensions, we will see that $c_{R}(4 D)$ will be fixed to some value less than 26. Therefore, for the mathematical consistency of the theory, we must require that the right moving sector contains an additional "internal" part which makes up for the difference, i.e. $c_{R}($ int $)+c_{R}(4 D)=26$. One of the aims of this paper is to compute $c_{R}($ int $)$ in each model and then find gauge symmetry groups that precisely give this value. This procedure will allow us to discover the gauge symmetries that are possible in these curved spacetime string models.

To construct a heterotic string we introduce four left moving coset fermions $\psi^{\mu}$ that are classified under $H$ as $G / H$ and form a $N=1$ supermultiplet together with the four bosons. The construction of the action that pocesses the superconformal symmetry is done along the lines of [6]. The left moving fermions $\psi^{\mu}$ are coupled to the gauge bosons in 
$H$. In the Hamiltonian language, the left moving stress tensor is expressed in the form of current algebra cosets [7] [8] as listed in Table-1, where $S O(3,1)_{1}$ represents the fermions.

This algebraic formulation allows an easy computation of the Virasoro central charges for left movers $c_{L}$ as well as the right movers $c_{R}(4 D)$. For a consistent theory we must set $c_{L}=15$. This condition puts restrictions on the various central extensions $k$ and/or background charges $Q_{0}, Q$, as listed in Table-2. After inserting these in $c_{R}(4 D)$ we find the deficit from the critical value of 26, i.e. $c_{R}(i n t)=26-c_{R}(4 D)$. As seen in the table, the resulting values for $c_{R}($ int $)$ fall within a narrow range. For case 2 or 3 it is possible to change the central charge within the range $11 \frac{1}{2}<c_{R}($ int $)<13$ by varying $k_{1}+k_{2}$. Similarly, the corresponding range for cases 9,10 is $12 \frac{1}{2}<c_{R}($ int $)<13$. For the remaining cases it is not possible to change $c_{R}($ int $)$ by using the remaining freedom with the $k^{\prime} s$.

\begin{tabular}{|c|c|c|c|}
\hline$\#$ & conditions for $c_{L}=15$ & $c_{R}(i n t)$ & gauge group, right movers \\
\hline 1 & $k=5$ & 11 & $\left(E_{7}\right)_{1} \times S U(5)_{1}$ \\
\hline 2 & $k_{1}-2=\frac{k_{2}-2}{2}\left(-1+\sqrt{\frac{3 k_{2}}{3 k_{2}-8}}\right)$ & $13-\delta$ & $\delta=\frac{12}{\left(k_{1}+k_{2}-4\right)\left(k_{1}+k_{2}-2\right)}$ \\
\hline 3 & $k=3$ & $11 \frac{1}{2}$ & $\left(E_{7}\right)_{1} \times S U(3)_{1} \times S U(2)_{2} \times U(1)_{1}$ \\
\hline 4 & $k=8 / 3$ & 13 & $\left(E_{8}\right)_{1} \times S O(10)_{1}$ \\
\hline 5 & $k_{1}=\frac{8 k_{2}-20}{3 k_{2}-8}, \quad k_{1}, k_{2}>\frac{8}{3}$ & 13 & $\left(E_{8}\right)_{1} \times S O(10)_{1}$ \\
\hline 6 & $k_{1}=\frac{8 k_{2}+20}{3 k_{2}+8}, k_{2}=1,2,3, \cdots$ & 13 & $\left(E_{8}\right)_{1} \times S O(10)_{1}$ \\
\hline 7 & $k=8 / 3$ & 13 & $\left(E_{8}\right)_{1} \times S O(10)_{1}$ \\
\hline 8 & $Q_{0}^{2}=\frac{3}{4}$ & 13 & $\left(E_{8}\right)_{1} \times S O(10)_{1}$ \\
\hline 9 & $Q_{0}^{2}=\frac{3}{4}+\frac{1}{2}\left(\frac{1}{k_{1}+2}+\frac{1}{k_{2}+2}-\frac{1}{k_{1}+k_{2}+4}\right)$ & $13-\delta$ & $\delta=\frac{12}{\left(k_{1}+k_{2}+4\right)\left(k_{1}+k_{2}+2\right)}$ \\
\hline 10 & $Q_{0}^{2}=\frac{3(k+3)}{4(k+2)},(e . g . \quad k=1)$ & $12 \frac{1}{2}$ & $\left(E_{8}\right)_{1} \times S U(3)_{1} \times S U(2)_{2} \times U(1)_{1}$ \\
\hline 11 & $Q_{0}^{2}=\frac{3 k+8}{4(k+2)}, k=1,2,3, \cdots$ & 13 & $\left(E_{8}\right)_{1} \times S O(10)_{1}$ \\
\hline 12 & $Q_{0}^{2}=\frac{3 k+8}{4(k+2)}, k=1,2,3, \cdots$ & 13 & $\left(E_{8}\right)_{1} \times S O(10)_{1}$ \\
\hline 13 & $Q_{0}^{2}=\frac{3 k-8}{4(k-2)}$ & 13 & $\left(E_{8}\right)_{1} \times S O(10)_{1}$ \\
\hline
\end{tabular}

At this point we mention the effect of a non-zero background charge $Q$ for the spacelike factor $\mathbb{R}$ in cases $2,3,4$ and 7 . The formula for $c_{R}(i n t)$ that is listed in the table for case 2 remains the same, but the conditions on the $k^{\prime} s$ change slightly. The new conditions take the same form as cases 9,10,11 and 12 as listed in the table respectively, except for the analytic continuations $Q_{0}^{2} \rightarrow-Q^{2}$ and $k_{i} \rightarrow-k_{i}$. However, since $0<Q^{2}<\frac{1}{12}$, the values of the new $k^{\prime} s$ in cases 2,5 must remain within a narrow range of those already fixed in 
Table-2. Only for cases 2,3,4 this has an effect on $c_{R}(i n t)$. For example, for case 3 we get $2.9 \leq k \leq 3$ (instead of $k=3$ ) and $11.24<c_{R}($ int $)<11 \frac{1}{2}$ (instead of $11 \frac{1}{2}$ ). The presence of a non-zero $Q$ does not change the discussion that follows.

The value of $c_{R}(i n t)=13$ that occurs for most of the cases is the same as the deficit for the popular heterotic string models that have four flat dimensions plus compactified dimensions described by a $c=9, N=2$ superconformal theory (i.e. $4+9+13=26$ ). Hence, for these cases, the appearance of $\left(E_{8}\right)_{1} \times S O(10)_{1}$ as the gauge group has precisely the same explanation as the usual approach. For the remaining cases we give an example of a gauge symmetry that will make up the deficit, as listed in Table- 2. Other gauge groups are clearly possible just on the basis of $c_{R}(i n t)$. For example, for case 1 one can have $S O(22)_{1}, \quad\left(E_{8}\right)_{1} \times S U(4)_{1}, \quad\left(E_{7}\right)_{1} \times S U(5)_{1}, \quad\left(E_{7}\right)_{1} \times S U(3)_{1} \times S U(2)_{1} \times$ $U(1)_{1}, \quad\left(E_{6}\right)_{1} \times S O(10)_{1}$, etc., as given in $[6]$.

The gauge symmetry is associated with a conformal theory of right movers. This additional part of the action may be constructed from right moving free fermions with appropriate boundary conditions, or by using other devices that are quite familiar. We can think of this part as another current algebra associated with the gauge group, and with the central extensions that are given in Table-2. This final step completes the action for the model. For the complete action for case 1, see [6]. Further discussion of the model is required to determine the symmetries consistent with modular invariance. At this stage it is encouraging to note that the desirable low energy symmetries, including $S U(3) \times S U(2) \times U(1)$, are contained in these curved space string models that have only four dimensions.

The special property of the models constructed in this paper is that they can be further investigated by using current algebra techniques. The simplest model is case 8 , since it is essentially flat, its quantum theory reduces to the manipulation of harmonic oscillators. For the remaining models the spectrum of low energy particles is obtained by computing the quadratic Casimir operators of the non-compact groups that define the model. The computation of the spectrum will be reported in a future publication. Since the flavor groups such as $S U(3) \times S U(2) \times U(1)$ or $S U(5), S O(10)$, etc. appear at level 1 , it is already evident that the quark and lepton type of matter will appear in triplets and doublets respectively.

An interesting question is how the repetition of the families will come about? In the traditional approach that includes compactified dimensions, the number of families is related to indices, such as Betti numbers, of the compactified space. In the present case, 
the four dimensional geometry has many new and interesting properties, such as duality, different topological sectors, etc. as seen in the global analysis of [3]. Therefore, we may expect that the repetition of families may have something to do with these properties. The repetition will show up in the algebraic approach by the number of distinct ways that it is possible to satisfy the on-mass-shell conditions for the same quark or lepton quantum number (e.g. different representations of the non-compact group). When this criterion is applied to the flat case 8 , we see that the presence of the background charge $Q_{0}$ allows two distict states to be associated with the same conformal dimension, thus leading to two families. Therefore, it is quite possible that the repetition of families may arise from the four dimensional geometry alone as described above.

We want to point out another possible source of family replication. It may be feasible to interpret part of the gauge group as a "family group", as it was done in the days before string theory. If this latter alternative is utilised for family replication, then one might quit the idea of "hidden sectors" attributable to groups such as $E_{8}$, and instead adopt a version of the gauge group which has complex representations. For example, in case 1, one of the possibilities that give $c_{R}($ int $)=11$ was $\left(E_{6}\right)_{1} \times S O(10)_{1}$, which has complex representations.

During the past year there has been many investigations [2-17] that explored the geometry of the sigma model-like action associated with some of these models. While the geometry for $d=2$ is interpreted as a black hole the singularity structure for $d=3,4$ is considerably more involved and interesting. For example, for $d=3$ a global analysis of the manifold shows that there are two topologically distinct sectors that can be pictured as the "pinched double trousers" or the "double saddle" [3]. Furthermore, the time dependent backgrounds that emerge allow for cosmological interpretations. These results were initially obtained at the semi-classical level using the lagrangian method (in patches of the geometry). More recently, fully quantum mechanical results were obtained by using conformally exact current algebra methods in a Hamiltonian formalism [4]. The algebraic method simultaneously yields the full global geometry, as was illustrated for $d=2,3,4$. Furthermore, the heterotic and type-II supersymmetric versions of these models were investigated and their conformally exact global geometry determined. By now the global geometry of all the above models have essentially been completed in [3] [4] [17]. The cases not covered directly in these references can be obtained with analytic continuation techniques. 
Are there additional models beyond the ones listed in the tables? Undoubtedly there are more, but they may not have the virtue of being solvable like the present ones that have a current algebra formulation. It is, of course, possible to imagine perturbations of the present models that may be formulated in the current algebra language and yield solvable cases. Such perturbations will tend to change the formulas for the central charges and it would be interesting to investigate how stable is $c_{R}(i n t)$ against these perturbations and how the gauge group is affected by them. On the basis of Table- 2 it seems that one cannot wander too far away from $c_{R}($ int $)=13$. Therefore the desirable low energy flavor symmetries are likely to remain. These are interesting questions that should be investigated.

We have argued that it is interesting to consider the possibility of heterotic string theories in only four spacetime dimensions and no additional compactified dimensions. This is possible only in curved spacetime, and such a string can be imagined to describe the very small distances or very early times in the Universe. 


\section{References}

[1] I. Bars and D. Nemeschansky, Nucl. Phys. 348 (1991) 89.

[2] E. Witten, Phys. Rev. D44 (1991) 314.

[3] I. Bars and K. Sfetsos, "Global Analysis of New Gravitational Singularities in String and Particle Theories", USC-92/HEP-B1 (hep-th/9205037), to appear in Phys. Rev. D (1992).

[4] I. Bars and K. Sfetsos, "Conformally Exact Metric and Dilaton in String Theory on Curved Spacetime", USC-92/HEP-B2 (hep-th 9206006), to appear in Phys. Rev. D (1992). ;

Konstadinos Sfetsos, "Conformally Exact Results for $S L(2, \mathbb{R}) \times S O(1,1)^{d-2} / S O(1,1)$ Coset Models", USC-92/HEP- S1, (hep-th 9206048)

[5] I. Bars, "Curved Space-Time Strings and Black Holes", in Proc. of XX ${ }^{\text {th }}$ Int. Conf. on Diff. Geometrical Methods in Physics, Eds. S. Catto and A. Rocha, Vol.2, p.695, (World Scientific, 1992).;

P. Ginsparg and F. Quevedo, "Strings on Curved Space-Times: Black Holes, Torsion, and Duality, LA-UR-92-640. ;

See also [3]

[6] I. Bars and K. Sfetsos, Phys. Lett. 277B (1992) 269.

[7] Y. Kazama and H. Suzuki, Nucl.Phys. B234 (1989) 232.

[8] I. Bars, Nucl. Phys. B334 (1990) 125.

[9] I. Bars and K. Sfetsos, Mod. Phys. Lett. A7 (1992) 1091.

[10] M. Crescimanno, Mod. Phys. Lett. A7 (1992) 489.

[11] J. B. Horne and G. T. Horowitz, Nucl. Phys. B368 (1992) 444.

[12] E. S. Fradkin and V. Ya. Linetsky, Phys. Lett. 277B (1992) 73.

[13] N. Ishibashi, M. Li, and A. R. Steif, Phys. Rev. Lett. 67 (1991) 3336.

[14] P. Horava, Phys. Lett. 278B (1992) 101.

[15] E. Raiten, "Perturbations of a Stringy Black Hole", Fermilab-Pub 91-338-T.

[16] D. Gershon, "Exact Solutions of Four-Dimensional Black Holes in String Theory", TAUP-1937-91.

[17] I. Bars and K. Sfetsos, $S L(2, \mathbb{R}) \times S U(2) / \mathbb{R}^{2}$ String Model in Curved Spacetime and Exact Conformal Results, USC-92/HEP-B3 (hep-th 920800x) 